\title{
DISTRIBUCIÓN CONOCIDA Y POTENCIAL DE LAS ESPECIES DE GEOTRUPINAE (COLEOPTERA: SCARABAEOIDEA) EN MÉXICO
}

\author{
Nuria TrotTA-MOREU1, Jorge M. LOBO ${ }^{2}$ \\ y Francisco J. CABRERo-SAÑUdo ${ }^{1}$ \\ 1Instituto de Ecología A.C., Departamento de Biodiversidad y Ecología Animal, \\ Km. 2.5 Carr. Antigua a Coatepec No. 351, Cong. El Haya, 91070, CP 91070 \\ Xalapa, Veracruz, MÉXICO. \\ 2Museo Nacional de Ciencias Naturales - CSIC, Departamento de Biodiversidad y Biología \\ Evolutiva. José Gutiérrez Abascal 2, CP 28006 Madrid, ESPAÑA. \\ nutrotta@gmail.com,mcnj117@mncn.csic.es, fcabrero@gmail.com
}

\section{RESUMEN}

Se recopiló la información disponible acerca de los geotrúpidos presentes en la República Mexicana, teniendo en cuenta las citas presentes en la literatura y en diversas colecciones entomológicas. A partir de estos datos se elaboró una base de datos (GEOMEX), la cual se utilizó, en primer lugar, para presentar un listado actualizado de todas las especies presentes en el territorio mexicano. También se elaboraron mapas de la distribución conocida de dichas especies, así como mapas que muestran la distribución potencial utilizando una metodología basada en modelos de máxima entropía (MaxEnt). Se presentan, así mismo, algunos datos biogeográficos así como los valores climáticos óptimos para cada especie.

Palabras Clave: Geotrupini, Bolboceratini, Athyreini, mapas de distribución, modelos predictivos, escarabajos del estiércol, México.

\begin{abstract}
The available data on the geotrupids species present in the Mexican Republic was compiled, taking into account the literature citations as well as the available data in several entomologic collections. A database (GEOMEX) was developed based on these data, which was useful to extract an actualized list of all the species present in the Mexican territory. Distribution maps, both observed and potential, were produced, those latter by means of a methodology based on maximum entropy models (MaxEnt). Some other biogeographic data are also supplied, as well as climatic optimum values for each species.

Key Words: Geotrupini, Bolboceratini, Athyreini, distribution maps, predictive models, dung beetles, Mexico.
\end{abstract}




\section{INTRODUCCIÓN}

El grado de conocimiento actual de los Geotrupinae a nivel taxonómico y faunístico en México está soportado, principalmente, por diversos trabajos realizados desde mediados del siglo pasado, en especial por Howden (1955, 1964, 2003, 2005), Halffter y Martínez (1962) y Zunino (1984). A partir de estos trabajos, actualmente es posible establecer un listado de las especies de Geotrupinae en México y de sus posibles distribuciones geográficas. Sin embargo, tal y como ocurre incluso en países en los que se ha realizado un esfuerzo de colecta mayor y más prolongado en el tiempo y, además, poseen una menor riqueza taxonómica (Dennis et al. 1999; Dennis \& Thomas 2000; Zaniewski et al. 2002; Reutter et al. 2003; Graham et al. 2004; Martínez-Meyer, 2005; Romo et al. 2006; Hortal et al. 2007; Lobo et al. 2007), la información corológica suele estar plagada de sesgos y únicamente permite esbozar una distribución general muy incompleta para la gran mayoría de las especies.

En la actualidad existen diversas herramientas estadísticas, las cuales, con el concurso de Sistemas de Información Geográfica, permiten elaborar hipótesis de distribución a partir de los datos biológicos disponibles y de la información sobre distintas variables ambientales que actúan como predictores (Guisan \& Zimmermann 2000). Evidentemente, la distribución real de los organismos esta influida por variables geográficas, históricas o por limitantes de dispersión (ver por ejemplo Pulliam, 2000), de modo que no todos los enclaves con condiciones climáticas o ambientales favorables son colonizados por las especies. Debido a ello y a la carencia de información fiable sobre la ausencia verdadera de las especies, las hipótesis de distribución generadas mediante estas técnicas de modelización suelen representar simulaciones de la distribución potencial de las especies (Soberón \& Peterson 2005; Peterson 2006). Es decir, mapas de idoneidad o probabilidad que indican la presencia de lugares favorables desde el punto de vista climático para la aparición de la especie.

Tras elaborar un listado actualizado de las especies de Geotrupinae presentes actualmente en México, poniendo al día la última revisión hecha por Howden (2003), el presente trabajo proporciona mapas individualizados para cada una de las especies de Geotrupinae presentes en México, en los cuales se incluye tanto la distribución conocida como la distribución potencial, generada mediante una de las técnicas de modelización actualmente considerada como fiable.

\section{MATERIAL Y MÉTODOS}

\section{Procedencia de los datos biológicos}

Se elaboró una base de datos recopilando toda la información disponible en la bibliografía, así como en diversos estudios no publicados a los que se ha tenido 
acceso (tesis de licenciatura, maestría y doctorado), destacando los trabajos de Howden (1955, 1964, 1974, 2003, 2005); Halffter \& Martínez (1962); y, Gómez \& López-Rojas (2004). También se consultaron diversas colecciones entomológicas, como las colecciones particulares de G. \& V. Halffter, E. Montes de Oca, F. Vaz de Mello, M. A. Morón, H. F. \& A. Howden, I. Martínez, L. Arellano, así como la colección del Instituto de Ecología A. C. (Xalapa, Veracruz, México)

La base de datos, que hemos nombrado GEOMEX, contiene hasta la fecha 1193 registros, correspondientes a 45 especies mexicanas de Geotrupinae (clasificación sensu Howden 2003; Cuadro 1). Aunque la taxonomía de alto rango para este grupo y sus relaciones filogenéticas internas no se encuentran aún bien establecidas (Howden 1982, Zunino 1984, Browne \& Scholtz 1995, 1999), el listado de especies puede considerarse fiable. En nuestro caso hemos incluido las nuevas especies descritas en los últimos años, completando así el catálogo proporcionado por la última revisión de Howden (2003) de las especies de Geotrupinae mexicanas. GEOMEX consta de 24 campos de información, pertenecientes a las siguientes categorías: a) campos taxonómicos (género y especie); b) campos geográficos (localidad, estado, altitud, latitud y longitud); c) campos relativos a la captura (día, mes y año, número de ejemplares capturados, método de captura, colector); $d$ ) campos eco-biológicos (sexo de los individuos, alimentación y hábitat); y, e) otros campos relacionados con el manejo ulterior de los ejemplares o de la información sobre estos (colección donde está depositado el ejemplar, persona que realizó la identificación, cita bibliográfica, otras observaciones). Tras georreferenciar las localidades en las que ha sido citada cada una de las especies (ver http://geonames.usgs.gov/gnishome.html) a una resolución de $0.08^{\circ}(25,080$ celdas para el territorio mexicano), se construyeron mapas de la distribución observada de cada una de las especies utilizando el programa Idrisi Kilimanjaro (Clark Labs. 2003).

\section{Datos climáticos utilizados}

Como predictores se utilizaron las diecinueve variables climáticas tomadas de la base de datos WorldClim (Hijmans et al. 2007): temperatura media anual, rango medio de temperaturas diurnas, isotermalidad, estacionalidad de temperaturas, temperatura máxima del mes más cálido, temperatura mínima del mes más frío, rango de temperaturas anuales, temperatura media del cuatrimestre más húmedo, temperatura media del cuatrimestre más seco, temperatura media del cuatrimestre más cálido, temperatura media del cuatrimestre más frío, precipitación anual, precipitación del mes más húmedo, precipitación del mes más seco, estacionalidad de las precipitaciones, precipitación del cuatrimestre más húmedo, precipitación del cuatrimestre más seco, precipitación del cuatrimestre más calido y precipitación del cuatrimestre más frío. 
Cuadro 1. Listado de las especies de Geotrupidae mexicanas según las dos clasificaciones taxonómicas principales comúnmente aceptadas: Howden (2003) en esta página y Zunino (1984) en la siguiente. Las especies marcadas con un asterisco no fueron asignadas a ningún género por Zunino (1984).

\section{Subf.Geotrupinae}

\section{Tribu Geotrupini}

Gen. Geotrupes

Subg. Megatrupes

cavicollis Bates, 1887

fisheri Howden, 1967

Subg. Onthotrupes

lobatus Howden, 1974

sobrinus Jekel, 1865

herbeus Jekel, 1865

viridiobscurus Jekel, 1865

truncaticornis Howden, 1864

sallei Jekel, 1865

nebularum Howden, 1964

guerreroensis Howden, 1964

pecki Howden, 1964

onitidipes Bates, 1887

Subg. Geohowdenius

cnephosus Howden, 1964

Subg. Halffterius

rufoclavatus Jekel, 1865

Subg. Haplogeotrupes

guatemalensis Howden, 1974

reddelli Howden, 1980

Gen. Ceratotrupes

fronticornis (Ericsson, 1847)

sturmi Jekel, 1865

bolivari Halffter \& Martínez, 1962

\section{Tribu Bolboceratini}

Gen. Bolbocerosoma

ritcheri Howden, 1955

pusillum Howden, 1955

confusum Brown, 1928

mexicanum Howden, 2005

Gen. Bolborhombus

sallei (Bates, 1887)

magnus (Howden, 1964)

parvulus Cartwright, 1953

nitidus Howden, 1964

Gen. Bolbocerastes

regalis Cartwright, 1953

serratus (LeConte, 1854)

imperials Cartwright, 1953

peninsularis (Schaeffer, 1906)

Gen. Bolbelasmus

arcuantus (Bates, 1887)

hornii (Rivers, 1886)

bajaensis Howden, 1964

rotundipennis Howden, 1964

variabilis Howden, 1964

Gen. Eucanthus

mexicanus Howden, 1964

impressus Howden, 1964

Tribu Athyreini

Gen. Neoathyreus

excavatus (Laporte de Castelnau, 1840)

fissicornis (Harold, 1880)

granulicollis Howden, 1964

hamifer (Boucomont, 1932)

interruptus Howden, 1964

mexicanus (Klug, 1845)

mixtus (LeConte, 1854) 
Fam. Geotrupidae

Subf. Geotrupinae

Tribu Geotrupini

Gen Megatrupes

cavicollis (Bates, 1887)

fisheri (Howden, 1967)

Tribu Ceratotrupini

Gen Onthotrupes

*lobatus (Howden, 1974)

sobrinus (Jekel, 1865)

herbeus (Jekel, 1865)

viridiobscurus (Jekel, 1865)

*truncaticornis (Howden, 1864)

*sallei (Jekel, 1865)

nebularum (Howden, 1864)

guerreroensis (Howden, 1864)

pecki (Howden, 1864)

onitidipes (Bates, 1887)

Gen. Geohowdenius

*cnephosus (Howden, 1864)

Gen. Halffterius

rufoclavatus (Jekel, 1865)

Gen. Haplogeotrupes

guatemalensis (Howden, 1974)

reddelli (Howden, 1967)

Gen. Ceratotrupes

fronticornis (Ericsson, 1847)

sturmi Jekel, 1865

bolivari Halffter \& Martínez, 1962

\begin{abstract}
Subf. Bolbocerinae
Tribu Bolboceratini

Gen. Bolbocerosoma

ritcheri Howden, 1955

pusillum Howden, 1955

confusum Brown, 1928

mexicanus Howden, 2005

Gen. Bolborhombus

sallei (Bates, 1887)

magnus (Howden, 1964)

parvulus Cartwright, 1953

nitidus Howden, 1964
\end{abstract}

Gen. Bolbocerastes

regalis Cartwright, 1953

serratus (LeConte, 1854)

imperiales Cartwright, 1953

peninsularis (Schaeffer, 1906)

Gen. Bolbelasmus

arcuantus (Bates, 1887)

hornii (Rivers, 1886)

bajaensis Howden, 1864

rotundipennis Howden, 1864

variabilis Howden, 1864

Gen. Eucanthus

mexicanus Howden, 1864

impressus Howden, 1864

Tribu Athyreini

Gen. Neoathyreus

excavatus (Laporte de Castelnau, 1840)

fissicornis (Harold, 1880)

granulicollis Howden, 1864

hamifer (Boucomont, 1932)

interruptus Howden, 1964

mexicanus (Klug, 1845)

mixtus (LeConte, 1854)

\section{Elaboración de las distribuciones potenciales}

La estimación de la distribución potencial se llevo a cabo mediante MaxEnt 2.2 (Maximum Entropy; Phillips et al. 2006) utilizando los parámetros definidos por defecto en el programa, pero incluyendo únicamente características lineares y cuadráticas. Entre 
las diversas técnicas disponibles, se ha elegido este método porque requiere únicamente información sobre la presencia de las especies y porque un reciente análisis comparado lo avala y recomienda (Elith et al. 2006). MaxEnt es un método de "aprendizaje automático" (machine learning) que busca encontrar la distribución más cercana a la homogeneidad (situación de entropía máxima en la que todos los valores tienen la misma probabilidad de ocurrir) pero restringiendo ésta según la información biológica disponible y las condiciones ambientales del área de estudio. El resultado es un mapa en el que cada celda posee un valor de favorabilidad que, oscilando entre 0 y 100 , es proporcional a la suma del valor de probabilidad de cada celda y de todas aquellas celdas con probabilidades iguales o menores a ella (Phillips et al. 2006).

\section{Validación de las distribuciones}

Estimar la capacidad de los modelos obtenidos para reflejar con fiabilidad la distribución de los organismos es un requisito esencial para que estas hipótesis sean útiles en conservación y biogeografía (Vaughan \& Ormerod 2005). Debido a la escasez de datos disponibles, la precisión de las hipótesis de distribución generadas fue estimada superponiendo cada uno de los modelos individuales obtenidos y calculando el número total de especies resultantes en distintos estados de México, cuyo inventario completo fue previamente estimado como fiable (Cuadro 2). Para calcular si el número conocido de especies en cada estado representa una estima fiel de la riqueza total existente, se calculó el estimador no paramétrico Chao2 (Colwell \& Coddington 1994), algoritmo que considerando el número de especies que aparecen en una o dos unidades de esfuerzo permite establecer la riqueza teórica total. En nuestro caso, se utilizó como medida de esfuerzo el número de registros existentes en GEOMEX de cada especie para cada estado de México (Hortal et al. 2006). Los cálculos fueron realizados mediante el programa EstimateS (Colwell 2000).

Como la superposición de los modelos individuales claramente genera sobrepredicciones en la riqueza total de especies (Cuadro 2), los mapas continuos de favorabilidad individuales fueron convertidos a mapas binarios (presencia-ausencia), eligiendo el punto de corte óptimo capaz de producir la mejor correlación entre los valores de riqueza observados y los valores de riqueza generados por la superposición de los modelos individuales. Dicho punto de corte se halló a una favorabilidad de 70 (coeficiente de correlación de Spearman entre la riqueza observada en cada estado bien muestreado y la riqueza predicha por superposición de los modelos individuales $=0.641, \mathrm{p}=0.003$ ). De este modo, los mapas que se presentan a continuación constituyen la hipótesis de distribución potencial consecuencia de la aplicación del mencionado punto de corte. En el caso de aquellas especies en las que la aplicación de dicho punto de corte impedía que todos los puntos de presencia observados estuviesen incluidos en la distribución potencial ( $\mathrm{n}=$ 17), se ha representado la distribución potencial predicha por MaxEnt aplicando 
como punto de corte el valor de favorabilidad mínimo encontrado en un punto de presencia. En el caso de aquellas especies con un único dato de distribución conocido (G. (Onthotrupes) lobatus, Bolbocerosoma mexicanus, Bolborhombus nitidus, Bolbelasmus horni, Bolbelasmus bajaensis y Eucanthus impressus), el modelo de distribución potencial que se presenta es el resultante de ampliar un 30\% los valores ambientales de las celdas en la que se observó la especie.

Cuadro 2. Número de especies observadas en cada estado Mexicano (SOBS), número de especies estimadas mediante el estimador no paramétrico Chao2 considerando el número de registros como medida de esfuerzo (SCHAO2), número de especies estimadas mediante la superposición de los modelos individuales de distribución generados con MaxEnt (SMAXENT) y número de especies resultante de aplicar el punto de corte sobre los valores de favorabilidad (entre 0 y 100) que permite obtener una mejor correlación con los valores de riqueza de especies observados (SOPTIMO; ver métodos). Sólo se incluyen los 19 estados cuyo número de especies observado es, al menos, el 50\% del estimado mediante Chao2.

\begin{tabular}{lcccc}
\hline Estado & $\mathbf{S}_{\text {OBS }}$ & $\mathbf{S}_{\mathbf{C H A O 2}}$ & $\mathbf{S}_{\mathbf{\text { MAXENT }}}$ & $\mathbf{S}_{\text {OPT IMO }}$ \\
\hline Baja California Sur & 3 & 3 & 23 & 10 \\
Chiapas & 7 & 7 & 26 & 12 \\
Chihuahua & 7 & 13 & 18 & 9 \\
Coahuila & 5 & 5 & 23 & 8 \\
Distrito Federal & 4 & 4 & 19 & 8 \\
Durango & 10 & 13 & 22 & 11 \\
Estado de México & 5 & 5 & 22 & 16 \\
Guerrero & 11 & 12 & 29 & 19 \\
Hidalgo & 10 & 17 & 25 & 9 \\
Jalisco & 11 & 11 & 28 & 12 \\
Michoacán de Ocampo & 10 & 11 & 29 & 17 \\
Morelos & 10 & 11 & 23 & 15 \\
Nayarit & 5 & 5 & 23 & 9 \\
Nuevo León & 9 & 11 & 22 & 9 \\
Oaxaca & 15 & 16 & 31 & 25 \\
Puebla & 10 & 10 & 31 & 23 \\
Sinaloa & 8 & 9 & 19 & 8 \\
Sonora & 7 & 7 & 14 & 9 \\
Veracruz & 14 & 15 & 24 & 16 \\
\hline
\end{tabular}




\section{RESULTADOS}

El sumatorio de los modelos de distribución obtenidos para las distintas especies y su comparación con los valores de riqueza de especies observados o estimados en cada estado demuestra que las áreas de distribución potencial obtenidas constituyen hipótesis de distribución que sobrepredicen la distribución real (Cuadro 2). A pesar de ello, se puede afirmar que los valores de riqueza de especies resultantes de aplicar el punto de corte óptimo siguen una pauta similar a la de los valores observados, no existiendo una sobrepredicción excesivamente acusada ni sesgada espacialmente (Fig. 1).

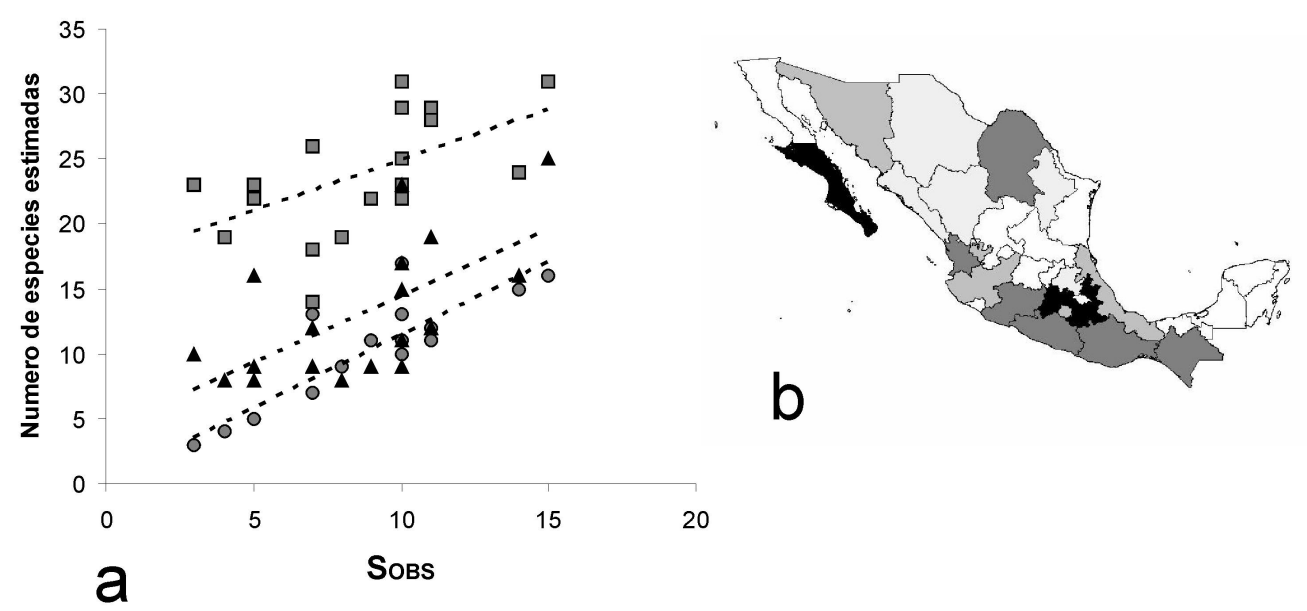

Figura 1. Relación entre el numero de especies observadas en diferentes estados mexicanos $\left(\mathrm{S}_{\mathrm{OBS}}\right)$ y el número de especies estimadas mediante Chao2 (ćrculos), considerando el número de registros como medida de esfuerzo, la superposición de los modelos individuales de distribución generados con MaxEnt (cuadrados) y el número de especies resultante de aplicar el punto de corte sobre los valores de favorabilidad (entre 0 y 100) que permite obtener una mejor correlación con los valores de riqueza de especies observados (triángulos). A la derecha se presentan los estados mexicanos con sobrepredicciones mayores del 100\% (negro), entre el 50\% y el 100\% (gris oscuro), sobrepredicciones menores del 50\% (gris claro) e infrapredicciones menores del 50\% (gris más claro). Sólo se incluyen los 19 estados cuyo número de especies observado es, al menos, el 50\% del estimado mediante Chao2. (ver Cuadro 2)

Los datos topográficos y climáticos de los puntos de presencia de cada una de las especies, así como el tamaño del área potencial de distribución aparecen en el Cuadro 3. Las especies de las tres principales tribus de Geotrupinae aparecen en diferentes condiciones ambientales. En lo que respecta a la altitud, la tribu Geotrupini se encuentra distribuida desde los 1500 y los 3200 metros. Sin embargo los Bolboceratini y Athyreini presentan una altitud media menor, casi desde el nivel del 
mar hasta los 1700 metros en la primera, y desde los 50 a los 1400 metros la segunda. Respecto a los valores climáticos, los Geotrupini se encuentran presentes en territorios con valores de precipitación mínima anual entre los 500 y los $2000 \mathrm{~mm}$, temperaturas mínimas de alrededor de $14^{\circ} \mathrm{C}$ y máximas de $22^{\circ} \mathrm{C}$. Por otra parte, los Bolboceratini se encuentran entre valores de precipitación mínima de $90 \mathrm{~mm}$ y máxima de $2500 \mathrm{~mm}$, temperaturas mínima de $17^{\circ}$ y máxima de $23^{\circ} \mathrm{C}$. Los Athyreini se encuentran presentes en enclaves con valores más altos de temperatura y precipitación que los otros dos grupos; precipitación mínima de $990 \mathrm{~mm}$, precipitación máxima de $2400 \mathrm{~mm}$, temperatura mínima de $17^{\circ}$ y máxima $24^{\circ} \mathrm{C}$ (Cuadro 3).

Estos datos muestran que los Geotrupini suelen vivir en localidades montañosas, más frías y con mayores precipitaciones que las especies de las otras tribus. Debido a ello, existiría un recambio altitudinal de especies, de manera que los Bolboceratini y Athyreini se encontrarían en altitudes bajas, bajo climas tropicales y áridos, mientras que los Geotrupini se hallarían en altitudes superiores, bajo climas templados o fríos. Esta ocupación de condiciones ambientales diferentes podría ser consecuencia del distinto origen biogeográfico de ambas subfamilias (Zunino 1984, Browne \& Scholtz 1995, 1999).

Cuadro 3. Tamaño del área de distribución potencial de cada especie $\left(\mathrm{en}^{\mathrm{km}}{ }^{2}\right)$, así como altitud media (en metros), precipitación (P) y temperatura medias (T) de las celdas de $0.08^{\circ}$ en las que se ha observado cada especie $( \pm \mathrm{SD})$. Los datos de altitud provienen de un modelo digital del terreno a una resolución de $1 \mathrm{Km}^{2}$ (ver United Status Geological Survey, 2006), mientras que los datos climáticos provienen de WorldClim (Hijmans et al. 2006). Como Geotrupes (Haplogeotrupes) reddelli está asociada a cuevas no hemos incluido datos ambientales para ella.

\begin{tabular}{lllll}
\hline Especies & Área & Altitud & T & P \\
\hline Geotrupes (Megatrupes) cavicollis & 17101 & 1695 & $17.9 \pm 0.90$ & $820 \pm 629$ \\
G. (Megatrupes) fisheri & 7275 & 2088 & $15.9 \pm 0.75$ & $1156 \pm 103$ \\
G. (Onthotrupes) lobatus & 247 & 1800 & 22.1 & 1727 \\
G. (Onthotrupes) sobrinus & 5526 & 1960 & $14.5 \pm 7$ & $1224 \pm 475$ \\
G. (Onthotrupes) herbeus & 4684 & 1869 & $14.8 \pm 8$ & $1033 \pm 949$ \\
G. (Onthotrupes) viridiobscurus & 3352 & 1973 & $15.4 \pm 3$ & $1357 \pm 936$ \\
G. (Onthotrupes) truncaticornis & 1388 & 2268 & $16 \pm 4$ & $1452 \pm 6$ \\
G. (Onthotrupes) sallei & 15116 & 1777 & $18.6 \pm 7$ & $1275 \pm 1052$ \\
G. (Onthotrupes) nebularum & 4847 & 1755 & $16.4 \pm 6$ & $1948 \pm 1803$ \\
G. (Onthotrupes) guerreroensis & 3843 & 1670 & $19.7 \pm 2$ & $1297 \pm 340$ \\
G. (Onthotrupes) pecki & 5254 & 1862 & $18.5 \pm 5$ & $1524 \pm 287$ \\
& & & & \\
\hline
\end{tabular}


Trotta-Moreu, Lobo \& Cabrero-Sañudo: Distribución de Geotrupinae en México

Cuadro 3. continúa

\begin{tabular}{|c|c|c|c|c|}
\hline Especies & Área & Altitud & $\mathbf{T}$ & $\mathbf{P}$ \\
\hline G. (Onthotrupes) onitidipes & 1900 & 1664 & $20.4 \pm 3$ & $2003 \pm 352$ \\
\hline G. (Geohowdenius) cnephosus & 10115 & 1962 & $13.5 \pm 3$ & $533 \pm 352$ \\
\hline G. (Halffterius) rufoclavatus & 4778 & 1955 & $15.4 \pm 11$ & $1105 \pm 725$ \\
\hline G. (Haplogeotrupes) guatemalensis & 903 & 2127 & $14 \pm 0.49$ & $1151 \pm 16$ \\
\hline G. (Haplogeotrupes) reddelli & - & - & - & - \\
\hline Ceratotrupes fronticornis & 18695 & 1615 & $18.5 \pm 12$ & $949 \pm 716$ \\
\hline Ceratotrupes sturmi & 12278 & 1693 & $20 \pm 2$ & $1033 \pm 598$ \\
\hline Ceratotrupes bolivari & 34050 & 1379 & $14.7 \pm 14$ & $1490 \pm 1597$ \\
\hline Bolbocerosoma ritcheri & 3161 & 669 & $21.3 \pm 1$ & $538 \pm 105$ \\
\hline Bolbocerosoma pusillum & 55916 & 1476 & $18.3 \pm 0.57$ & $324 \pm 12$ \\
\hline Bolbocerosoma confusum & 5094 & 379 & $21.8 \pm 1$ & $685 \pm 103$ \\
\hline Bolbocerosoma mexicanus & 2871 & 191 & $22.6 \pm 3$ & $758 \pm 107$ \\
\hline Bolborhombus sallei & 90678 & 1126 & $20.4 \pm 7$ & $845 \pm 868$ \\
\hline Bolborhombus magnus & 300228 & 621 & $21.1 \pm 2$ & $453 \pm 313$ \\
\hline Bolborhombus parvulus & 12292 & 237 & $21.7 \pm 3$ & $273 \pm 187$ \\
\hline Bolborhombus nitidus & 405 & 600 & $27.6 \pm 138$ & $777 \pm 389$ \\
\hline Bolbocerastes regalis & 5579 & 168 & $21.5 \pm 0.57$ & $136 \pm 88$ \\
\hline Bolbocerastes serratus & 93137 & 979 & $20.9 \pm 3$ & $998 \pm 998$ \\
\hline Bolbocerastes imperialis & 181998 & 531 & $20.8 \pm 4$ & $437 \pm 449$ \\
\hline Bolbocerastes peninsularis & 11905 & 210 & $21.6 \pm 3$ & $255 \pm 221$ \\
\hline Bolbelasmus arcuatus & 31473 & 899 & $23.1 \pm 6$ & $2483 \pm 2495$ \\
\hline Bolbelasmus horni & 885 & 500 & 18.4 & 188 \\
\hline Bolbelasmus rotundipennis & 229363 & 1427 & $20 \pm 4$ & $1410 \pm 1129$ \\
\hline Bolbelasmus variabilis & 37462 & 1192 & $20.3 \pm 7$ & $1191 \pm 1254$ \\
\hline Bolbelasmus bajaensis & 1133 & 9 & $21 \pm 3$ & $92 \pm 13$ \\
\hline Eucanthus mexicanus & 61671 & 1732 & $17.4 \pm 7$ & $1288 \pm 1291$ \\
\hline Eucanthus impressus & 6007 & 1207 & $17.2 \pm 2$ & $228 \pm 33$ \\
\hline Neoathyreus excavatus & 68316 & 854 & $20 \pm 11$ & $1620 \pm 1656$ \\
\hline Neoathyreus fissicornis & 11907 & 1079 & $20.1 \pm 8$ & $1655 \pm 1364$ \\
\hline Neoathyreus granulicollis & 38477 & 1442 & $17 \pm 9$ & $1059 \pm 1067$ \\
\hline Neoathyreus hamifer & 815 & 54 & $24.4 \pm 0.92$ & $3154 \pm 133$ \\
\hline Neoathyreus interruptus & 47164 & 762 & $21.2 \pm 9$ & $2083 \pm 1698$ \\
\hline Neoathyreus mexicanus & 79477 & 923 & $22.6 \pm 7$ & $2373 \pm 2651$ \\
\hline Neoathyreus mixtus & 164100 & 750 & $21 \pm 6$ & $995 \pm 773$ \\
\hline
\end{tabular}




\section{Distribución de los Geotrupini}

\section{Subgénero Megatrupes}

Este subgénero, endémico de México, está formado por dos especies con distribuciones disyuntas. Geotrupes cavicollis (Fig. 2a) se encuentra distribuida en el Noroeste de México, en la Sierra Madre Occidental, mientras que G. fisheri (Fig. 2b) se distribuye por el Sistema Volcánico Transversal. Geotrupes cavicollis parece ocupar lugares más cálidos y secos con una temperatura media de $17.9{ }^{\circ} \mathrm{C}$ y una precipitación media de $820 \mathrm{~mm}$, mientras que Geotrupes fisheri habitaría localidades algo más frías y húmedas con una temperatura media de alrededor de $16^{\circ} \mathrm{C}$ y una precipitación media alrededor de los $1150 \mathrm{~mm}$ (Cuadro 3).

Las distribuciones potenciales obtenidas para las dos especies amplían el rango de distribución conocido en ambas especies. Así, G. cavicollis también poseería condiciones ambientales adecuadas en la Sierra Madre Oriental y algunas zonas del Altiplano hacia la costa del Pacífico. Geotrupes fisheri podría también extender su distribución hacia algunos puntos de la Sierra Madre del Sur.
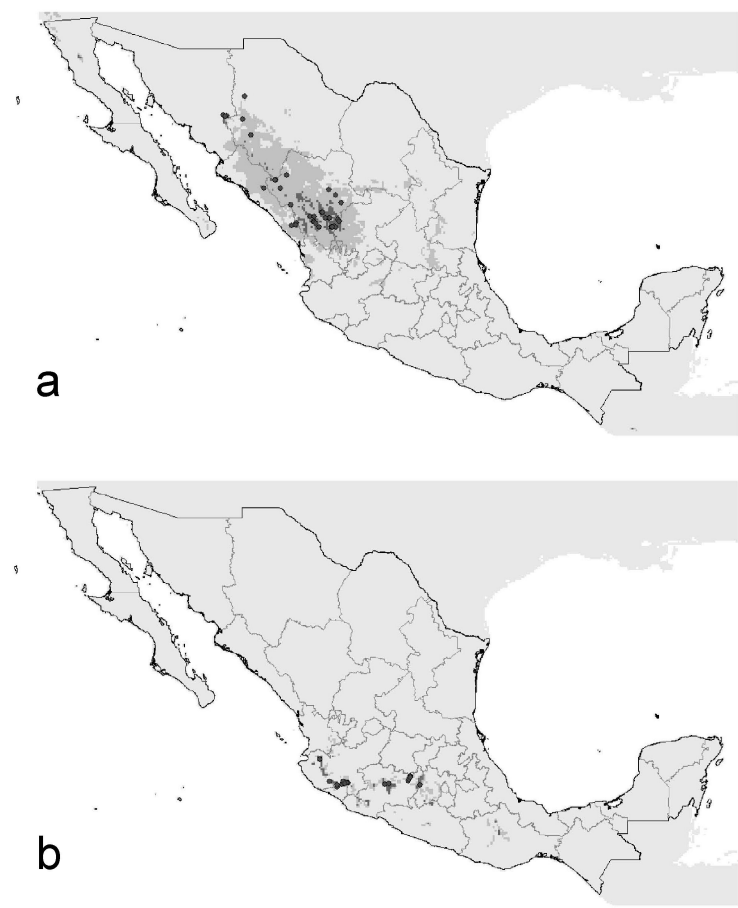

Figura 2. Mapas de distribución de las especies del subgénero Megatrupes. Los puntos en negro representan la distribución observada, mientras que la distribución potencial estimada mediante el punto de corte óptimo se representa en gris oscuro y la distribución potencial con el valor de favorabilidad mínimo encontrado en los lugares con presencia en gris claro. a) G. cavicollis; b) G. fisheri. 


\section{Subgénero Onthotrupes}

Este subgénero es, básicamente, endémico de la República Mexicana, salvo por la especie G. onitidipes, que se extiende también hacia Guatemala y El Salvador (Fig. 3a). Las especies del subgénero Onthotrupes presentan en general distribuciones restringidas que apenas se solapan entre sí. En general, su distribución se constriñe a la Sierra Madre del Sur y al Sistema Volcánico Transversal, habitando localidades con temperaturas medias que oscilan entre los $14^{\circ}$ y $\operatorname{los} 22^{\circ} \mathrm{C}$ y precipitaciones medias entre los 1000 y los $2000 \mathrm{~mm}$ (Cuadro 3).

Se pueden discriminar tres grupos de especies de Onthotrupes diferentes, según la localización de su distribución. Un primer grupo, constituido únicamente por $G$. onitidipes, se distribuiría por la Sierra de Chiapas y Guatemala (Fig. 3a). Un segundo grupo (G. pecki, G. guerreroensis y $G$. truncaticornis) estaría formado por aquellas especies distribuidas principalmente por la Sierra Madre del Sur, aunque G. pecki también se encontraría presente en la Sierra de Chiapas (Fig.3b, c y d). Geotrupes lobatus, especie conocida únicamente de una localidad de la Sierra de Oaxaca (Fig. 3e), podría encontrase también en zonas cercanas a ésta, según sugiere la distribución potencial realizada. Por último, un tercer grupo de Onthotrupes estaría formado por aquellas especies que presentan distribuciones en el Sistema Volcánico Transversal (G. viridiobscurus, $G$. herbeus, G. sobrinus, G. sallei y G. nebularum); éstas son las que, en general, muestran rangos de distribución conocidos y potenciales más amplios. Geotrupes viridiobscurus se distribuye por la Sierra Madre del Sur, sugiriendo su distribución potencial una ampliación de su rango a diversos puntos interiores de esta Sierra (Fig. 3f). Geotrupes herbeus y G. sobrinus se distribuyen por el Sistema Volcánico Transversal, siendo su localización observada muy restringida (Fig. 3g, h). Sin embargo, en ambas especies la distribución potencial amplia su área de distribución hacia la Sierra Madre del Sur y la Sierra de Chiapas. Geotrupes sallei y G. nebularum son las especies con una distribución más amplia. La primera se distribuye por la Sierra Madre del Sur y el Sistema Volcánico Transversal; la distribución potencial amplia su rango hacia la Sierra de Chiapas (Fig. 3i). Geotrupes nebularum se distribuye por la Sierra Madre Oriental, el Sistema Volcánico Transversal y la Sierra Madre del Sur. Los mapas de distribución potencial también amplían la presencia de esta especie a diversos puntos del interior de estas sierras (Fig. 3j).

\section{Subgénero Geohowdenius}

Éste es un subgénero neártico (Howden 2003), que presenta una sola especie en México, $G$. cnephosus, con una distribución bastante restringida hacia zonas con precipitaciones bajas (alrededor de $500 \mathrm{~mm})$ y temperaturas templado-frías $\left(13^{\circ} \mathrm{C}\right.$; Cuadro 3). Tanto su área de distribución potencial, como su distribución conocida, corresponden a la parte norte de la Sierra Madre Occidental (Fig. 4). Esta especie está muy relacionada con G. opacus Haldeman 1853 y G. egeriei Germar, 1824, que presentan una amplia distribución en los Estados Unidos.

\section{Subgénero Halffterius}

Este subgénero esta compuesto por una única especie, G. rufoclavatus (Fig. 5), endémica de México. Su distribución conocida se limita al Sistema Volcánico Transversal, habitando praderas o claros de bosque de pinos, encinos y oyameles situados a partir de los $1900 \mathrm{~m}$ (Howden 2003), en localidades con una temperatura media de unos $15^{\circ} \mathrm{C}$ y una precipitación media de $1100 \mathrm{~mm}$ (Cuadro 3). La hipótesis de distribución potencial generada extiende su presencia hasta la Sierra Madre Oriental, la Sierra Madre del Sur y Chiapas. 

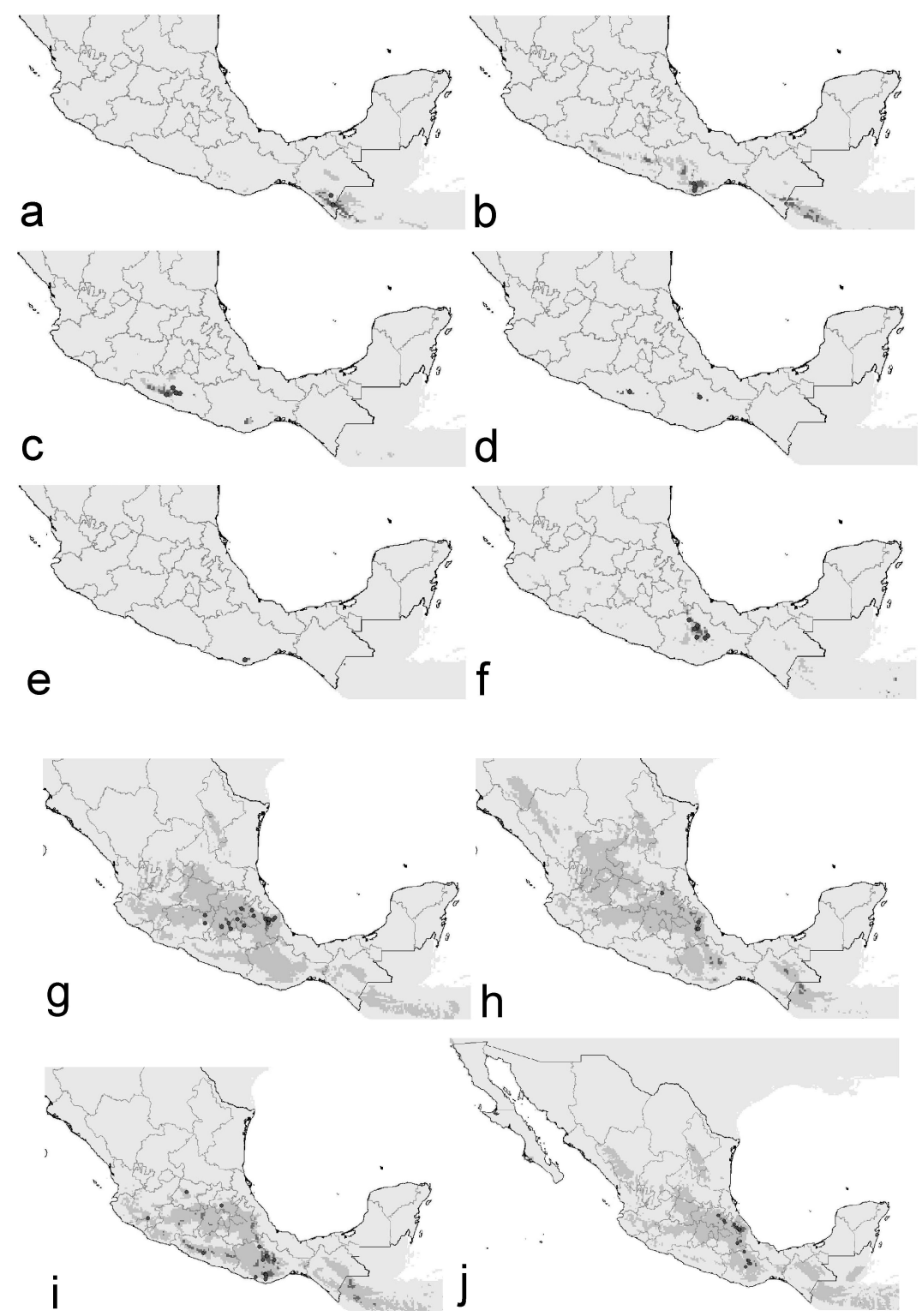

Figura 3. Mapas de distribución de las especies del subgénero Onthotrupes (ver Figura 2). a) G. onitidipes; b) G. pecki; c) G. guerreroensis; d) G. truncaticornis; e) G. lobatus; f) G. viridiobscurus; g) G. herbeus; h) G. sobrinus; i) G. sallei; j) G. nebularum. 
Trotta-Moreu, Lobo \& Cabrero-Sañudo: Distribución de Geotrupinae en México

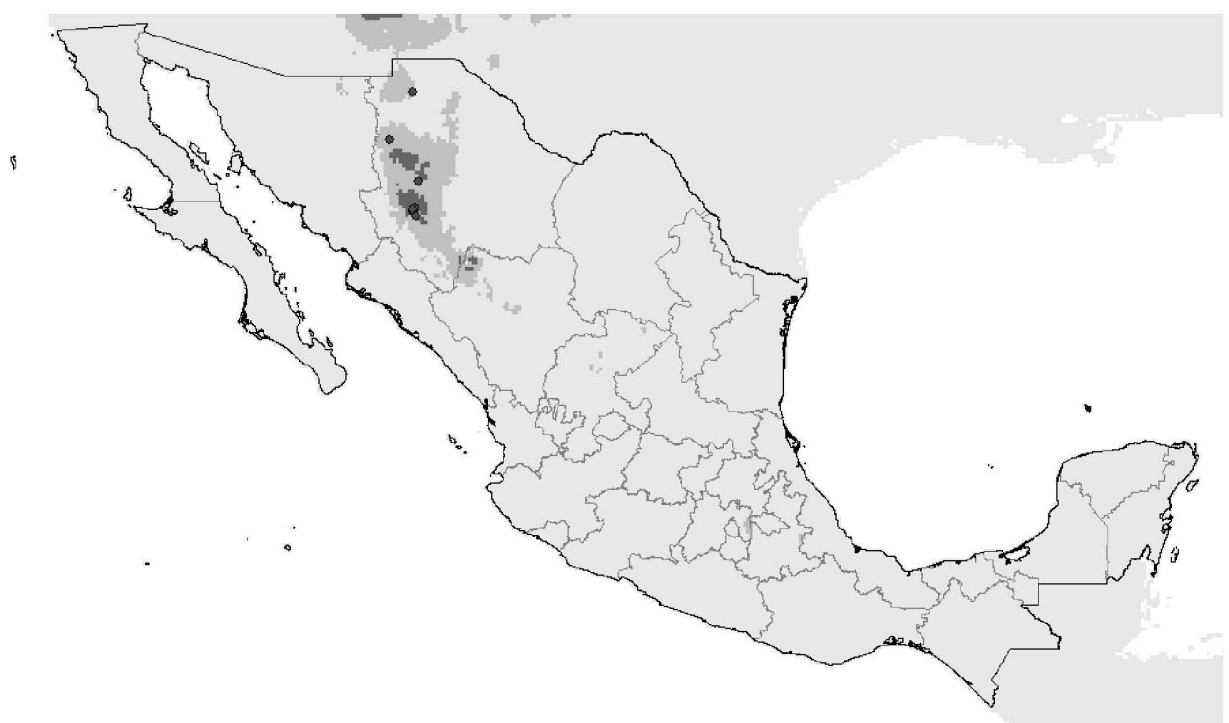

Figura 4. Mapa de distribución de G. (Geohowdenius) cnephosus.

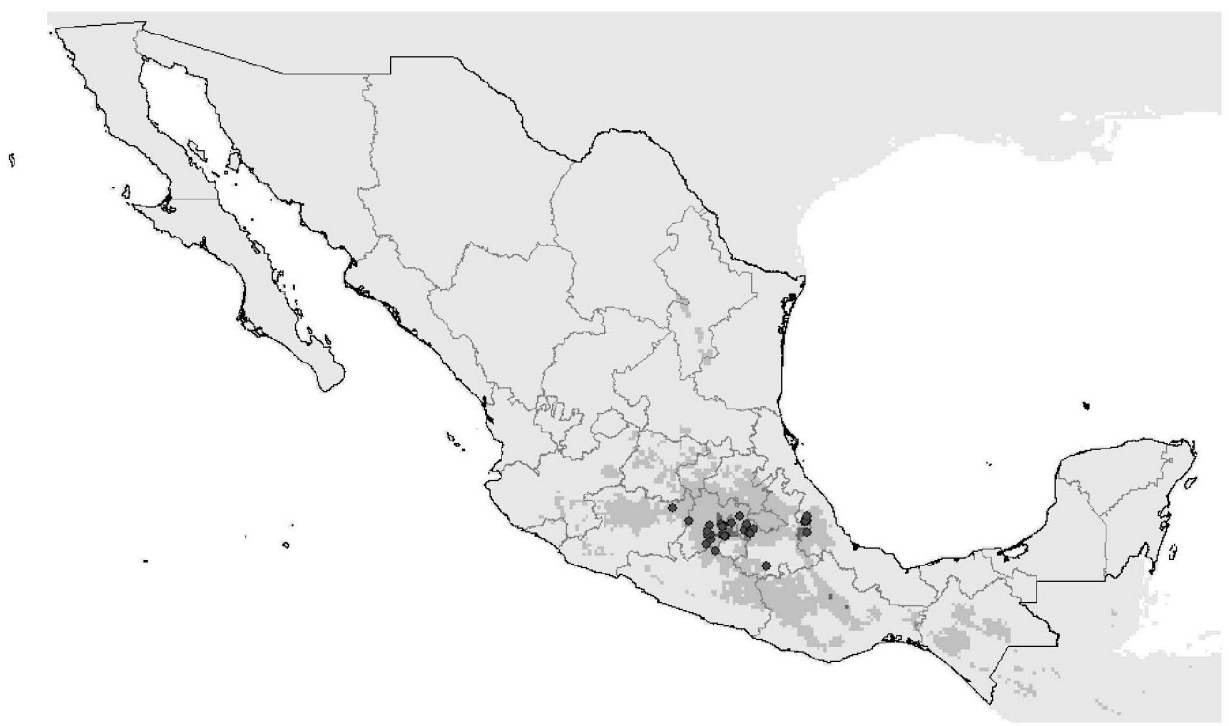

Figura 5. Mapa de distribución de G. (Halffterius) rufoclavatus. 


\section{Subgénero Haplogeotrupes}

Al igual que el resto de Geotrupini, Haplogeotrupes es un subgénero neártico. Los mapas de distribución potencial de las especies de este subgénero coinciden con los de su distribución conocida. En el caso de G. redelli no se han elaborado mapas de distribución potencial ya que, al tratarse de una especie troglobia, sería erróneo estimar su distribución en base a variables climáticas del medio exterior. Únicamente ha sido citada de las cuevas de Tasalolpan y Xochitlan en el estado de Puebla. En el caso de G. guatemalensis solamente se conocen citas muy puntuales del Estado de Chiapas (Fig. 6), en concreto de la localidad de San Cristóbal de las Casas, aunque su distribución se extiende hacia Guatemala. Los mapas de distribución potencial para esta especie muestran escasas localidades adicionales muy cercanas a la localidad de colecta.

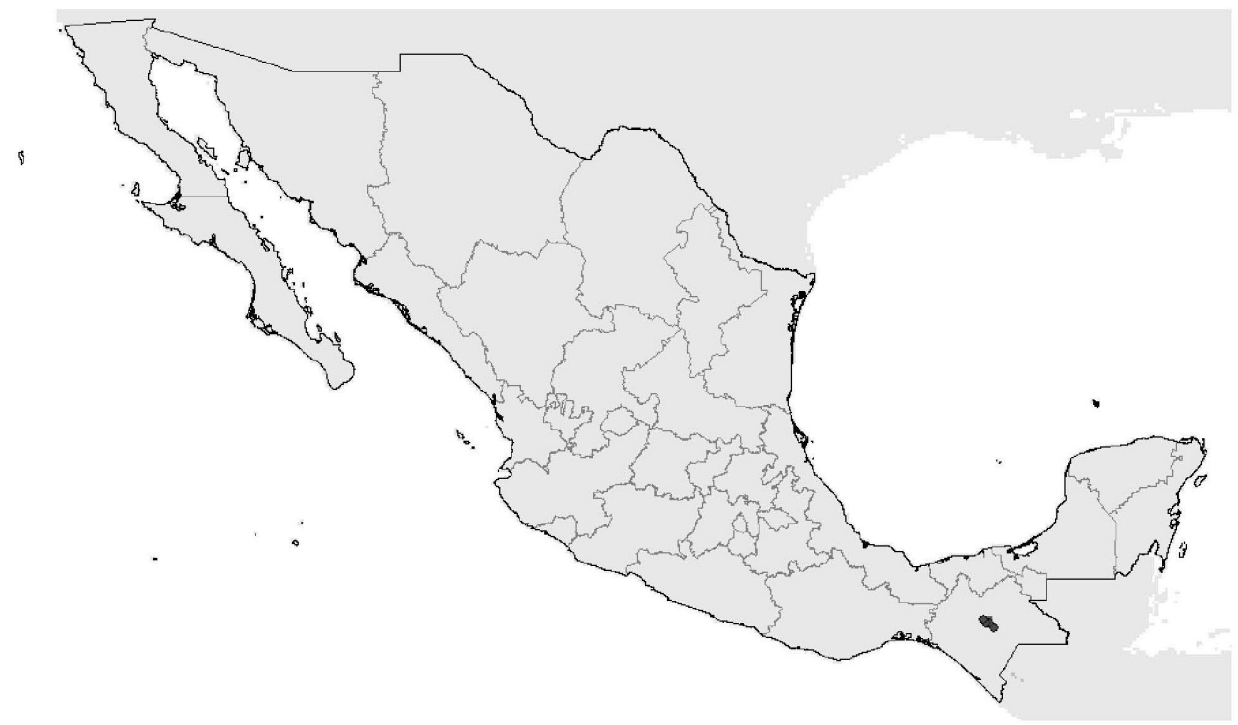

Figura 6. Mapas de distribución del género G. (Haplogeotrupes) guatemalensis.

\section{Género Ceratotrupes}

Se trata de un género actualmente endémico de México de origen neártico (Halffter \& Martínez 1962, Halffter 1987), cuyas especies poseen distribuciones extensas y, por tanto, gradientes ambientales amplios (entre los 14 y $20^{\circ} \mathrm{C}$ de temperatura y los 950 y $1500 \mathrm{~mm}$ de precipitación) (Cuadro 3). Las tres especies de Ceratotrupes se distribuyen principalmente por el Sistema Volcánico Transversal, la Sierra Madre Occidental y la Sierra Madre del Sur, a altitudes que oscilan entre los 1300 y los 1600 metros de altitud.

Los mapas potenciales obtenidos indican que las tres especies de este género solaparían sus áreas de distribución en los estados de Oaxaca y Guerrero, extendiéndose también por Chiapas 
y Guatemala, aunque no se han observado individuos de Ceratotrupes al este del estrecho de Tehuantepec. Ceratotrupes bolivari (Fig. 7a) es la especie del género que presenta una distribución más amplia llegando hasta el norte de México. Ceratotrupes fronticornis (Fig. 7b) también se distribuye por el norte de México, aunque parece ser capaz de habitar en el occidente del país. Por último, C. sturmi (Fig. 7c) es la especie que presenta una distribución más restringida, habitando únicamente diversas áreas de los estados de Oaxaca y Guerrero.
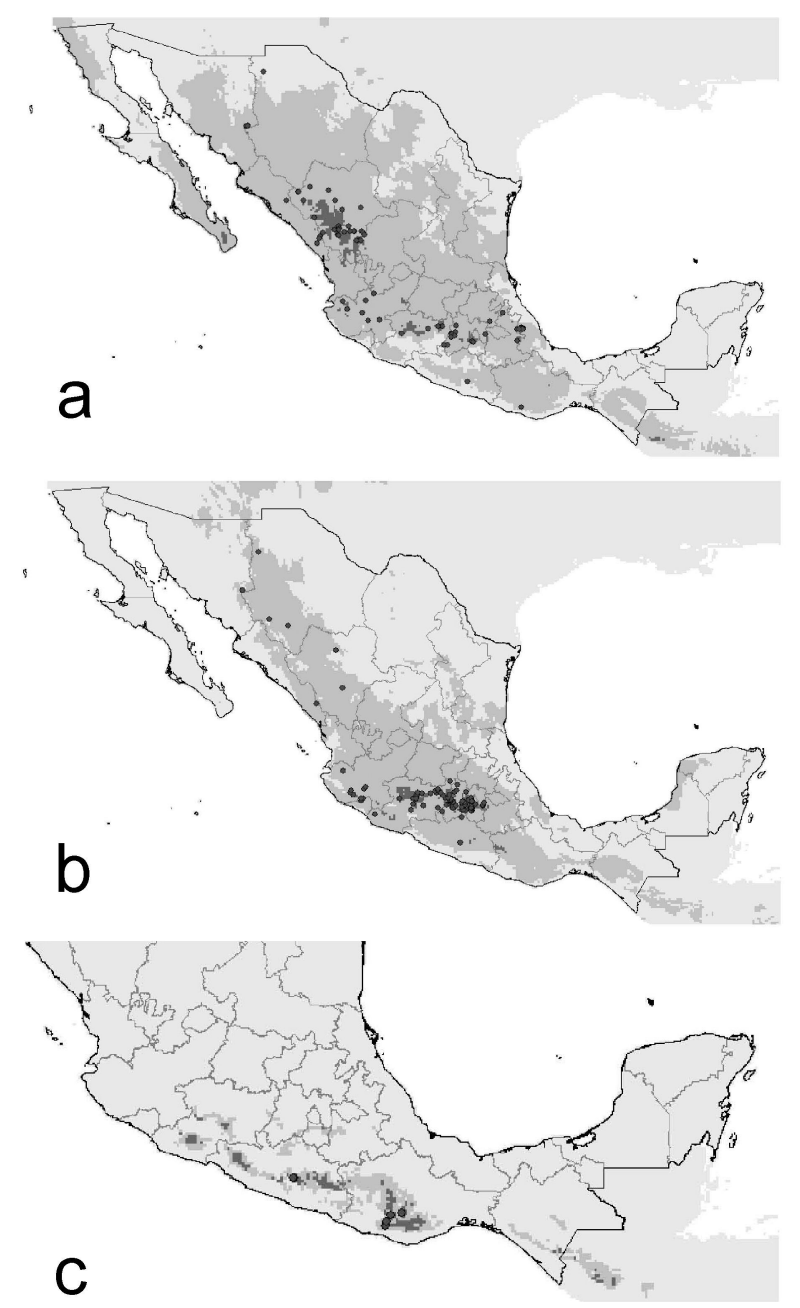

Figura 7. Mapas de distribución del género Ceratotrupes. a) C. bolivari; b) C. fronticornis; c) C. sturmi. 


\section{Distribución de los Bolboceratini mexicanos}

\section{Género Bolbocerosoma}

Este género neártico se distribuye en la República Mexicana por el norte de la Sierra Madre Oriental y el Altiplano Mexicano, desde los 200 a los $1500 \mathrm{~m}$ de altitud, áreas con temperaturas medias que oscilan entre $\operatorname{los} 18^{\circ} \mathrm{C}$ y $\operatorname{los} 22^{\circ} \mathrm{C}$ y precipitaciones medias entre los $300 \mathrm{~mm}$ y los $750 \mathrm{~mm}$. (Cuadro 3). De las cuatro especies mexicanas, B. pusillum es la que presenta una distribución más amplia, extendiéndose a lo largo de todo el Altiplano Mexicano hasta la Sierra Madre Occidental (Fig. 8a). Su distribución potencial amplia su presencia en diversos puntos del Altiplano Mexicano. El resto de las especies que forman este género ( $B$. ritcheri, B. confusum y B. mexicanum) son especies con distribuciones conocidas y potenciales muy restringidas (la última incluso endémica del país), situadas casi exclusivamente en el norte de la Sierra Madre Oriental (Fig. 8b, c y d).
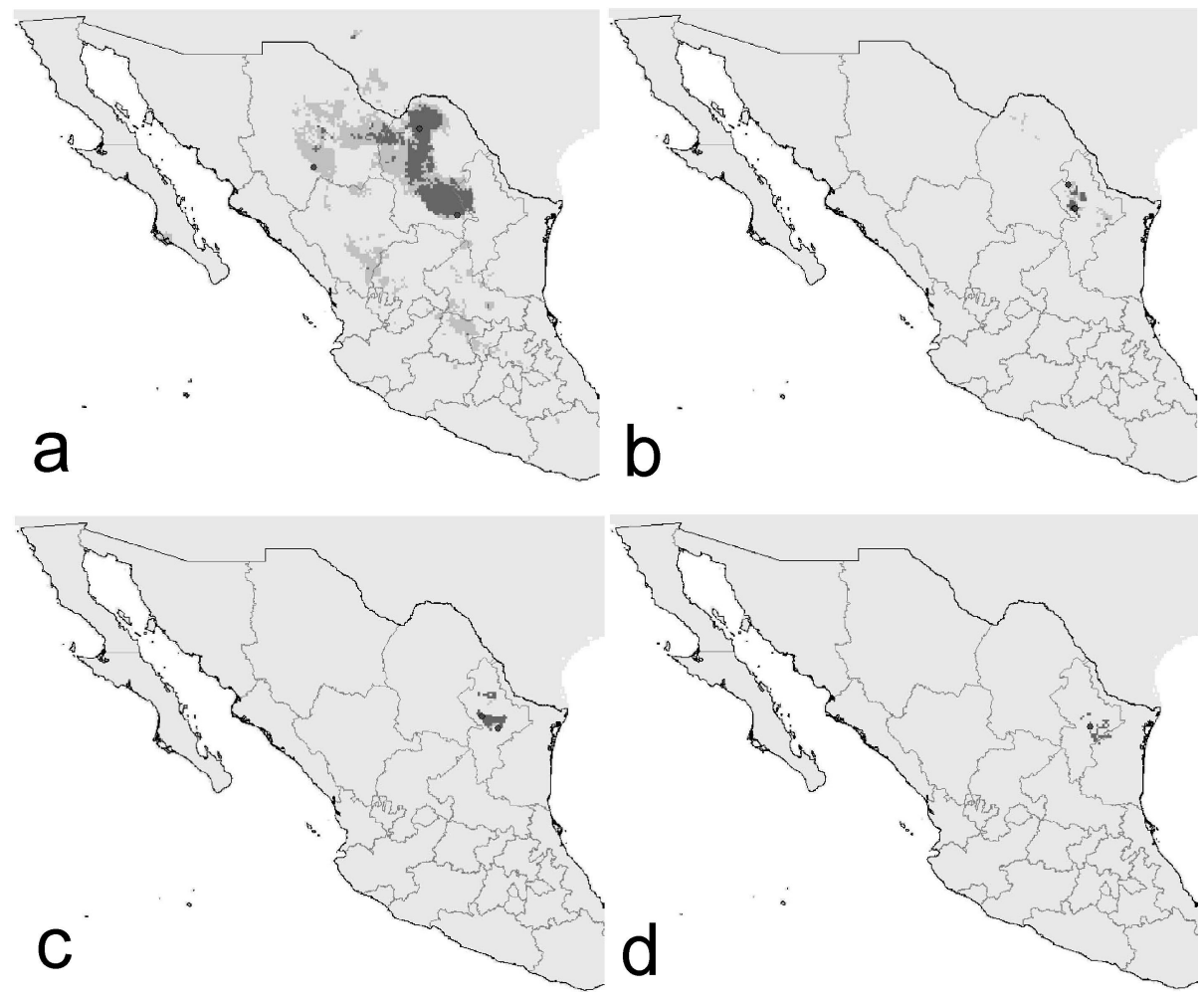

Figura 8. Mapas de distribución del género Bolbocerosoma. a) B. pusillum; b) B. ritcheri; c) B. confusum; d) B. mexicanus. 


\section{Género Bolborhombus}

Todas las especies de este género de distribución neártica se encuentran presentes en México, habitando localidades desde los 200 a los $1100 \mathrm{~m}$ de altitud con temperaturas medias entre los $20^{\circ}$ y los $27^{\circ} \mathrm{C}$ y precipitaciones que oscilan entre los 270 y los $850 \mathrm{~mm}$ (Cuadro 3)

Bolborhombus sallei y B. magnus muestran distribuciones amplias, estando presente la primera en casi todo el territorio mexicano, salvo en algunas zonas del Golfo de México y de la Península de Yucatán. Los datos de distribución potencial para B. sallei amplían su presencia en zonas de la costa oeste (Fig. 9a). Bolborhombus magnus se distribuye por el Altiplano Mexicano y el Sistema Volcánico Transversal; su mapa potencial amplia su rango hasta Baja California, Sonora y la Depresión del Balsas (Fig. 9b). Bolborhombus parvulus y B. nitidus presentan distribuciones mucho más restringidas; la primera se distribuye por Baja California, Sinaloa y Sonora, y la segunda en Michoacán (Fig. 9c, d). Las distribuciones potenciales para estas dos especies no amplían apenas su rango de distribución, salvo algunos puntos dispersos en Baja California y en Michoacán para B. nitidus.

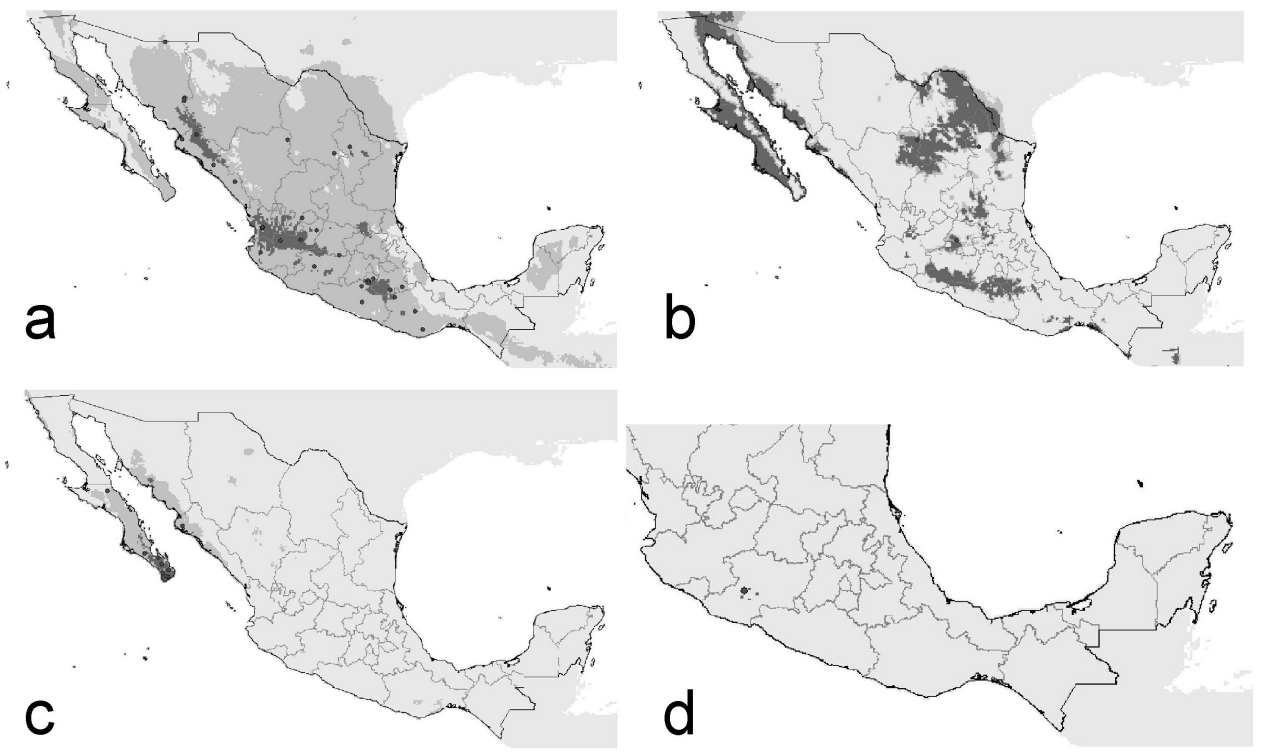

Figura 9. Mapas de distribución del género Bolborhombus. a) B. sallei; b) B. magnus; c) B. parvulus; d) B. nitidus.

\section{Género Bolbocerastes}

Este es otro género neártico presente en la Zona de Transición Mexicana, habitando localidades entre los $160 \mathrm{y}$ los 1000 metros con temperaturas medias en torno a los $20^{\circ} \mathrm{C} \mathrm{y}$ precipitaciones entre 130 y $250 \mathrm{~mm}$ (Cuadro 3). La mayor parte de las especies de este género 
se encuentran distribuidas por la mitad occidental de México, mientras que sólo una especie habita en la mitad oriental. Así, B. serratus se distribuye principalmente por el noreste de México, aunque potencialmente podría también estar presente por toda la costa oriental, sobre todo hacia el estado de Tamaulipas (Fig. 10a). Por el contrario, B. regalis, B. peninsularis y $B$. imperialis aparecen principalmente en la zona noroccidental de México (estados de Baja California, Sonora y, para la última, también Chihuahua). Bolbocerastes imperialis presenta la distribución más extensa, ampliando la distribución potencial su presencia hasta el Altiplano y la Depresión del Balsas (Fig. 10b). Bolbocerastes peninsularis ha sido colectada en Baja California y su distribución potencial sólo amplia en diversos puntos su rango conocido (Fig. 10c). Por último, B. regalis se distribuye por los estados de Baja California Norte y Sonora. El mapa de distribución potencial sugiere que la distribución de esta especie podría ampliarse a otras localidades de la misma región (Fig. 10d).
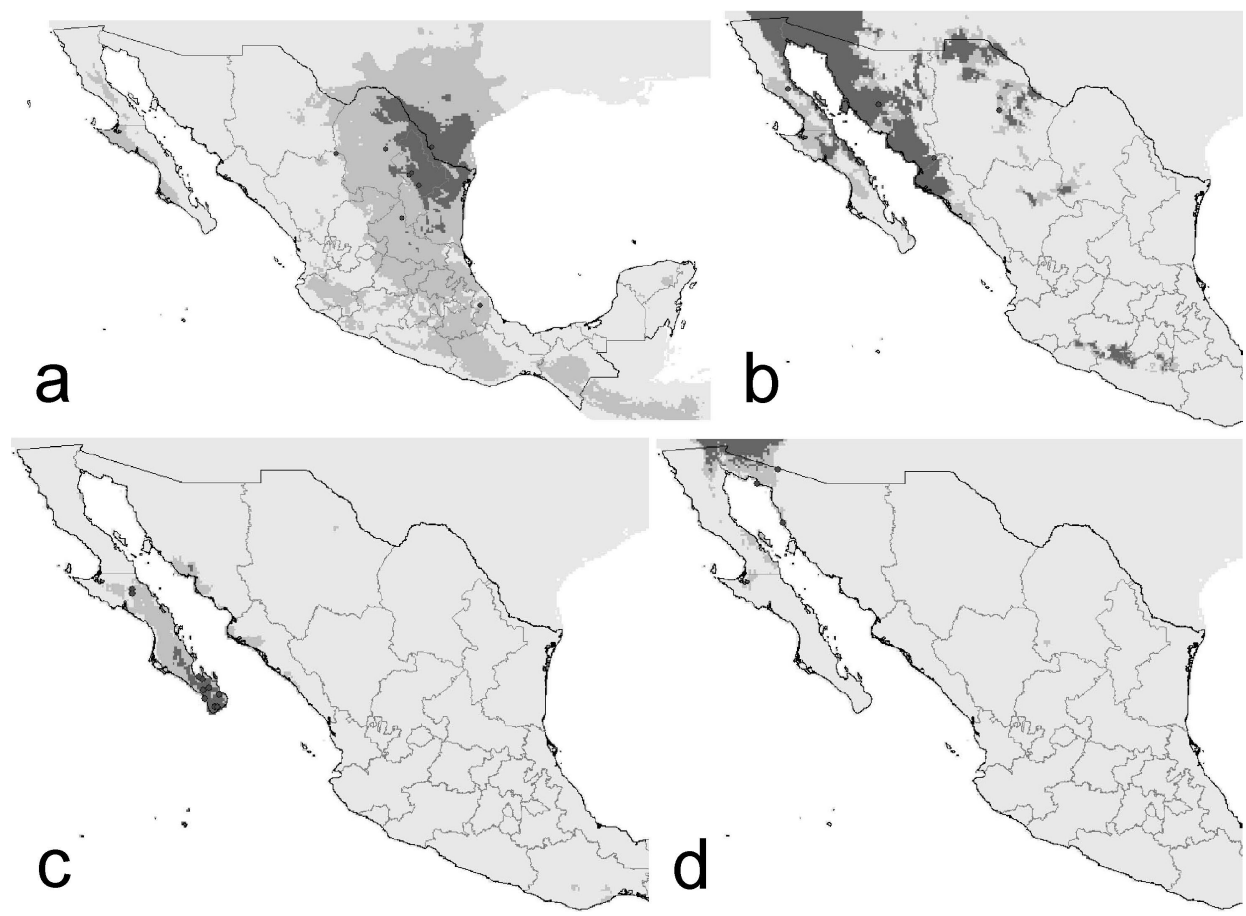

Figura 10. Mapas de distribución del género Bolbocerastes. a) B. serratus; b) B. imperialis; c) B. peninsularis; d) B. regalis.

\section{Género Bolbelasmus}

El género Bolbelasmus presenta una distribución bastante amplia, pues se extiende por las regiones Paleártica, Oriental, Neártica y Neotropical (Howden 2003). Cinco especies se encuentran presentes en México, de las cuales tres son endémicas. Pueden hallarse 
desde los 900 a los 1500 metros de altitud media, en lugares con temperaturas medias que oscilan entre los $18^{\circ} \mathrm{C}$ y $\operatorname{los} 23^{\circ} \mathrm{C}$ y precipitaciones medias entre los 90 y los $2500 \mathrm{~mm}$. (Cuadro 3).

Bolbelasmus arcuatus es una especie de distribución básicamente Neotropical, que se extiende en la República Mexicana por la región central y meridional, mostrando una distribución potencial que amplia notablemente su área conocida (ver Fig. 11a). B. variabilis es la especie mexicana que presenta una distribución más amplia, incluyendo la región Neártica y la Neotropical, y siendo más abundante hacia la costa oeste, donde se ampliaría su distribución potencial (Fig. 11b). Bolbelasmus rotundipennis se distribuye exclusivamente por la zona central de México, aunque su distribución potencial muestra que su rango podría extenderse hacia las costas occidentales y orientales, la Sierra de Chiapas y Guatemala (Fig. 11c). Por último, B. bajaensis y B. horni presentan distribuciones muy restringidas en Baja California; de $B$. horni solamente se conoce una cita puntual de la localidad de Ensenada (Fig. 11d) y de B. bajaensis de la localidad de Mulege (Fig. 11e). Los mapas potenciales apenas amplían su distribución conocida como consecuencia de la escasez de datos.
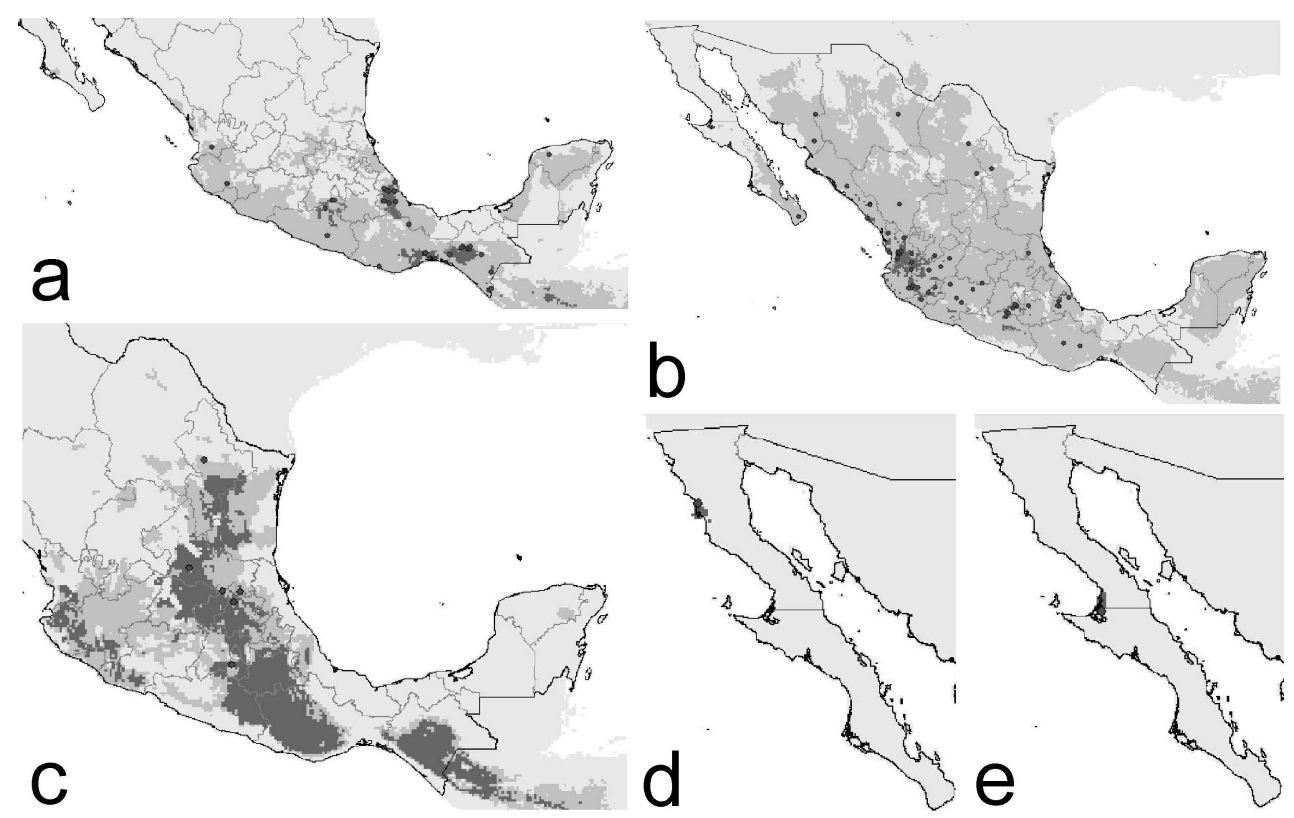

Figura 11. Mapas de distribución del género Bolbelasmus. a) B. arcuatus; b) B. variabilis; c) $B$. rotundipenis; d) B. horni; e) B. bajaensis. 


\section{Género Eucanthus}

Este género posee una amplia distribución, incluyendo las regiones Neártica, Neotropical y Australiana (Howden 2003), aunque en México solo pueden encontrarse dos especies, una de ellas endémica (E. mexicanus) distribuida por el Sistema Volcánico Transversal y la Sierra Madre Occidental. La distribución potencial de E. mexicanus sugiere una extensión de área conocida en el Sistema Volcánico Transversal y también en la Sierra de Chiapas (Fig. 12a). Eucanthus impressus se extiende principalmente por el norte de México mostrando una distribución observada y potencial restringida al norte del estado de Chihuahua (Fig. 12b). Ambas especies se localizan en enclaves con temperaturas medias en torno a los $17^{\circ} \mathrm{C}$, aunque E. mexicanus habita localidades con mayores precipitaciones medias $(1280 \mathrm{~mm})$ que $E$. impressus (230 mm; Cuadro 3).
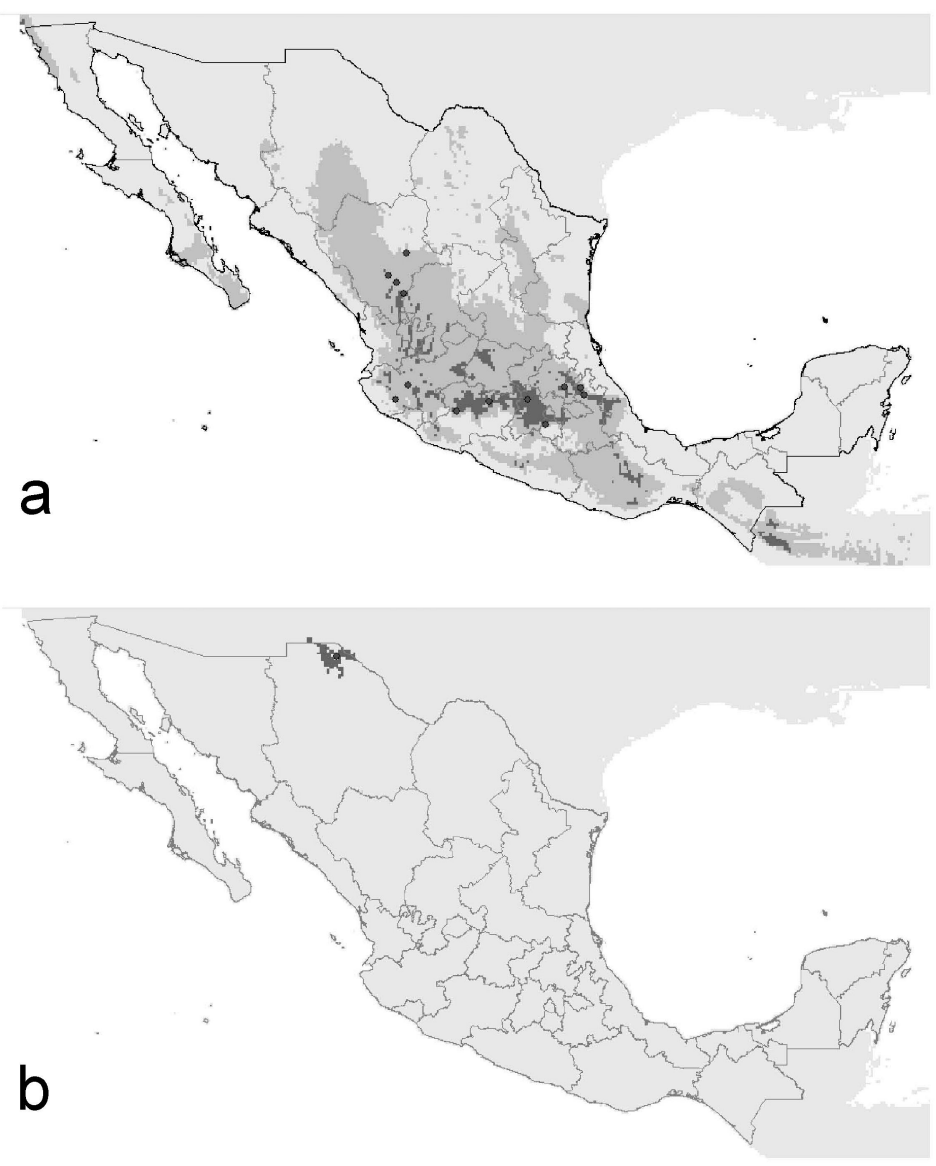

Figura 12. Mapas de distribución del género Eucanthus. a) E. mexicanus; b) E. impresus. 


\section{Distribución de los Athyreini mexicanos}

Esta tribu esta distribuida por las regiones Neotropical, Afrotropical y Oriental (Howden 2003), existiendo un único género en México, Neoathyreus, el cual posee una distribución típicamente Neotropical.

\section{Género Neoathyreus}

El género Neoathyreus presenta un gran número de especies en Sudamérica (Howden 1985); en México, la mayoría de las especies se distribuyen por las regiones centrales y meridionales en altitudes que oscilan entre los 50 y los 1400 metros de altitud, temperaturas medias que varían entre los 17 y $24^{\circ} \mathrm{C}$, y precipitaciones medias que fluctúan entre los 990 y $3200 \mathrm{~mm}$ (Cuadro 3). Neoathyreus fissicornis, N. granulicollis y N. mixtus son especies que alcanzan latitudes superiores a las de las demás especies y presentan una distribución más extensa en el territorio mexicano. Neoathyreus granulicollis y N. mixtus son las únicas especies endémicas del género; mientras $N$. mixtus parece distribuirse por la costa oriental
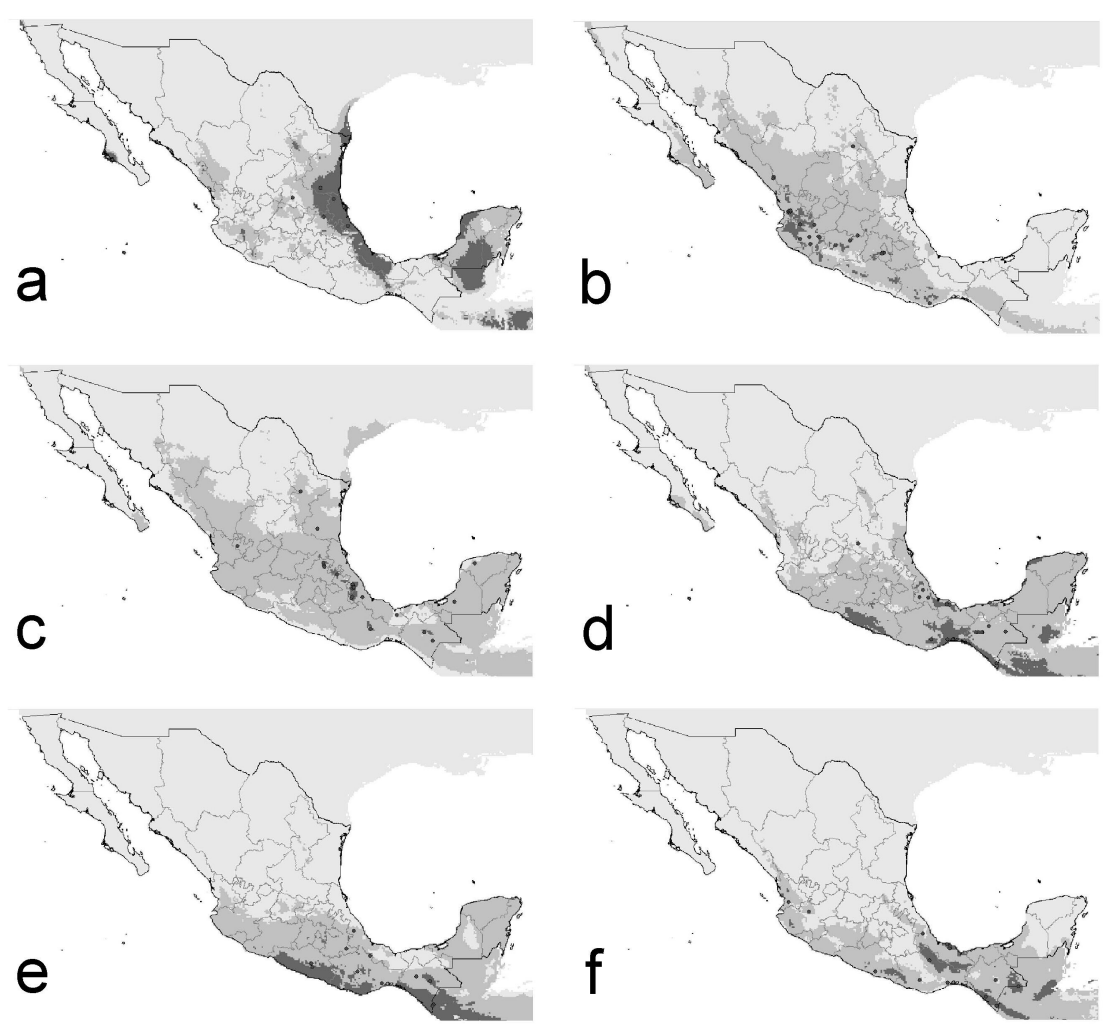

Figura 13. Mapas de distribución del género Neoathyreus. a) N. mixtus; b) N. granulicollis; c) N. fissicornis; d) N. excavatus; e) N. mexicanus; f) N. interruptus; g) N. hamifer. 
(Fig. 13a), N. granulicollis se extiende hacia la mitad occidental (Fig. 13b). Las áreas de distribución potencial para $N$. mixtus amplían su rango conocido hacia la costa oriental y los estados de Yucatán, Campeche y Quintana Roo. El área de distribución potencial de $N$. granulicollis amplia notablemente su rango hacia el este y el noroeste del país. Algo similar ocurre con $N$. fissicornis, especie colectada en diversas localidades alejadas del centro y sur de México que, potencialmente, podría habitar extensas áreas del país, excepto la región más septentrional (Fig. 13c).

El resto de las especies de este género presentes en México (N. excavatus, $N$. mexicanus, $N$. interruptus y $N$. hamifer) poseen una distribución más meridional, apenas sobrepasando las distribuciones potenciales el Sistema Volcánico Transversal (Fig. 13d, e, f, g). Sólo N. hamifer, únicamente citada del estado de Veracruz, muestra una distribución potencial restringida.

\section{DISCUSIÓN}

Cuando los datos conocidos de distribución de las especies son escasos y sesgados, la aplicación de modelos predictivos permite realizar distribuciones hipotéticas de utilidad en conservación y biogeografía. Ello es así, porque sus resultados son reproducibles y coherentes, al estar basados en las condiciones climáticas del territorio de interés. Estos mapas, no sólo nos proporcionan una imagen razonable de la distribución de las especies, sino que constituyen una herramienta útil para el diseño y planificación de nuevas colectas, sobre todo en el caso de regiones poco muestreadas.

Los mapas así generados deben considerarse una aproximación al conocimiento de la distribución potencial de los organismos, en el hipotético caso de que los factores capaces de restringir el rango de distribución no operasen (factores históricos, interacciones bióticas o limitantes de dispersión). Las distribuciones potenciales que hemos presentado podrían considerarse imágenes que nos muestran, en general, cuáles serían las tendencias de distribución de estas especies si a lo largo de la historia evolutiva de estos taxones no hubiera habido ninguna otra influencia que la climática. Sin embargo, la fauna de Geotrupinae mexicana está compuesta por grupos taxonómicos con diferentes orígenes e historias biogeográficas, los cuales han marcado la distribución actual de sus especies. El conocimiento de esta información biogeográfica complementa y da sentido a las distribuciones presentes en los mapas.

Así, según se ha sugerido, la tribu Geotrupini habría penetrado en América siguiendo un patrón de dispersión Neártico (Halffter 1976). Los antecesores de esta tribu habrían colonizado este continente a través del estrecho de Bering a partir del Plioceno, utilizando probablemente como corredores para su dispersión los sistemas orográficos occidentales de la región Neártica, ya que estas especies poseen una mayor afinidad por las zonas de montaña y los climas templado-fríos; estas zonas podrían haber favorecido también su aislamiento tras el último periodo de glaciación Pleistocénica. 
Para los Bolboceratini, existen actualmente dos teorías acerca de su origen. Howden (1982) propone que los Bolboceratini puedan ser, junto con Lethrini y Athyreini, el grupo hermano de los demás Geotrupinae; Zunino (1984) también admite esta hipótesis y sugiere una cladogénesis mesozoica que habría dado lugar a la vicarianza geográfica de un grupo Laurásico, anterior a los actuales Geotrupini, y otro Gondwánico, del que procederían los Bolboceratini. Por su parte, Browne \& Scholtz $(1995,1999)$ no aceptan una relación próxima entre estas dos tribus, puesto que sitúan a los Bolboceratini como el grupo hermano de la familia Pleocomidae. En cualquiera de los casos, según muestran nuestros mapas, la mayor parte de los Bolboceratini mexicanos poseen una afinidad hacia enclaves con temperaturas cálidas y precipitaciones moderadas, lo cual correspondería a un linaje típico con un patrón Paleoamericano, cuya entrada en Norteamérica se habría producido a partir del Cretácico (Halffter 1976). Esto coincide con Howden (2003), que opina que las especies mexicanas de Bolboceratini tendrían un origen totalmente Laurásico.

Dentro de Bolboceratini, los Eucanthus merecen una mención especial, puesto que, aunque actualmente se encuentran incluidos dentro de esta tribu, serían en realidad el grupo hermano natural del resto de Bolboceratini y Athyreini (Browne 1991; Verdú et al. 2004). Para Eucanthus se han observado en las Américas dos linajes con distribuciones diferentes, Neártica y Neotropical/Australasiática, respectivamente (Browne 1991), desconociéndose si el origen de ambos linajes es idéntico (ambos Gondwánicos) o diferente (uno Gondwánico y otro Laurásico). Los mapas conocidos y predichos nos ofrecen distribuciones muy diferentes para las dos especies de este género. De este modo, hasta no confirmarse filogenéticamente las relaciones de las especies incluidas dentro de Eucanthus, no puede aventurarse nada sobre la región de origen de las especies presentes en México.

En cuanto a los Athyreini, la mayor parte de los autores los consideran el grupo hermano de los Bolboceratini (Howden 1982; Browne 1991; Browne \& Scholtz 1995, 1999). Según Halffter (1987), la colonización mexicana de este linaje se habría producido durante el Plio-Pleistoceno desde la región Neotropical. Este posible origen coincide con el hecho de que presenten una afinidad hacia climas tropicales, de que, en efecto, existan muchas más especies en Sudamérica y de que se distribuya también por la región Afrotropical y Oriental (Howden 2003). Muy probablemente, esto esté indicando que el origen de los Athyreini sea Gondwánico.

\section{AGRADECIMIENTOS}

Agradecemos a Gonzalo Halffter, Henry Howden, Miguel Ángel Morón, Fernando Vaz de Mello, Imelda Martínez, Enrique Montes de Oca, Lucrecia Arellano y al Instituto de Ecología por permitirnos examinar sus colecciones, por el material proporcionado y por sus acertados comentarios. 
Este trabajo ha sido posible gracias a una beca de investigación del Ministerio de Asuntos Exteriores y de Cooperación (MAE-AECI) de España concedida a NT-M, y una beca postdoctoral del Ministerio de Educación y Ciencia de España concedida a FJC-S.

\section{LITERATURA CITADA}

Browne, D. J. 1991. Wing structure of the genus Eucanthus Westwood; confirmation of the primitive nature of the genus (Scarabaeoidea: Geotrupidae; Bolboceratinae). The Journal of the Entomological Society of Southern Africa. 54(2): 221-230.

Browne, D. J. \& C. H., Scholtz. 1995. Phylogeny of the families of the Scarabaeoidea (Coleoptera) based on characters of the hindwing articulation, hindwing base and wing venation. Systematic Entomology. 20: 145-173.

. 1999. A phylogeny of the families of Scarabaeoidea (Coleoptera). Systematic Entomology. 24: 51-84.

Clark Labs. 2003. Idrisi Kilimanjaro. Gis software package. Clark Labs, Worcester, M.A.

Colwell, R. K. \& J. A. Coddington. 1994. Estimating terrestrial biodiversity through extrapolation. Philosophical Transactions of the Royal Society of London. Series Biological Sciences. 345: 101-118.

Colwell, R. K. 2000. EstimateS: Statistical Estimation of Species Richness and Shared Species from Samples (Shoftware and User's Guide), version 6.0. Disponible en http://viceroy.eeb.uconn.edu/estimates.

Dennis, R.L.H., T.H. Sparks, \& P.B., Hardy. 1999. Bias in butterfly distribution maps: the effects of sampling effort. Journal of Insect Conservation. 3: 33-42.

Dennis, R.L.H. \& C.D., Thomas. 2000. Bias in butterfly distribution maps: the influence of hot spots and recorder's home range. Journal of Insect Conservation. 4: 73-77.

Elith J., Graham C.H., Anderson R.P., Dudík M., Ferrier S., Guisan A., Hijmans R.J., Huettmann F., Leathwick J.R., Lehmann A., Li J., Lohmann L.G., Loiselle B.A., Manion G., Moritz C., Nakamura M., Nakazawa Y., Overton J.M., Peterson A.T., Phillips S.J., Richardson K., Scachetti-Pereira R., Schapire R.E., Soberón J., Williams S., Wisz M.S., Zimmermann N.E. 2006. Novel methods improve prediction of species' distributions from occurrence data. Ecography. 29: 129-151.

Gómez, B. \& López-Rojas. 2004. Primer registro de Geotrupes (Onthotrupes) onitidipes Bates, 1887 para México (Coleoptera: Scarabaeidae: Geotrupidae). Entomotropica. Boletín de Entomología Venezolana. 19: 47-48.

Graham, C.H., S. Ferrier, F. Huettman, C. Moritz, \& A.T. Peterson. 2004. New developments in museum-based informatics and applications in biodiversity analysis. Trends in Ecology and Evolution. 19: 497-503.

Guisan, A. \& N.E. Zimmermann. 2000. Predictive habitat distribution models in ecology. Ecological. Modelling. 135: 147-1

Halffter, G. 1976. Distribución de los insectos en la zona de transición mexicana. Relaciones con la entomofauna de Norteamérica. Folia Entomológica Mexicana. 35: 1-64

Halffter, G. 1987. Biogeography of the Montane Entomofauna of México and Central America. Annual Reviews of Entomology. 32: 97-114 
Halffter, G. \& A. Martínez. 1962. Monografía del Género Ceratotrupes Jekel (Coleopt. Scarab. Geotrup.). Ciencia. 11(4): 145-159.

Halffter, V., Y. López-Guerrero \& G. Halffter. 1985. Nesting and ovarian development in Geotrupes cavicollis Bates (Coleoptera: Scarabaeidae). Acta Zoológica Mexicana. 7:1-28.

Hijmans, R. J., S. Cameron, J. Parra, P. Jones, A. Jarvis \& K. Richardson. 2006. WorldClim version 1.4. Museum of Vertebrate Zoology of the University of California, CIAT, and Rainforest CRC. http://www.worldclim.org/.

Hortal, J., P. A. V. Borges \& C. Gaspar. 2006. Evaluating the performance of species richness estimators: sensitivity to sample grain size. Journal of Animal Ecology. 75: 274-287.

Hortal, J., J.M. Lobo \& A. Jiménez-Valverde. 2007. Limitations of biodiversity databases: case study on seed-plant diversity in Tenerife (Canary Islands). Conservation Biology. 21(3): 853-863.

Howden, H. F. 1955. Biology and taxonomy of North American beetles of the Subfamily Geotrupinae with revisions of the Genera Bolbocerosoma, Eucanyhus, Geotrupes and Peltotrupes (Scarabaeidae). Proceedings of the United States National Museum. 104: 151319.

. 1964. The Geotrupinae of North and Central America. Memoirs of the Entomological Society of Canada. 39: 1-91.

. 1974. Additional records and descriptions of North and Central American Geotrupinae (Coleoptera, Scarabaeidae). Canadian Journal of Zoology. 52: 567- 573. . 1982. Larval and adult characters of Frickius Germain, its relationship to the Geotrupini, and a phylogeny of some mayor taxa in the Scarabaeoidea (Insecta: Coleoptera). Canadian Journal of Zoology. 60: 2713-2724.

- 1985. A revision of the Southamerican species in the genus Neoathyreus Howden and Martínez (Coleoptera: Scarabaeidae, Geotrupinae). Contributions of the American Entomological Institute. 21: 1-95.

. 2003. Geotrupinae [95-106]. In: Morón M. A. (Editor), Atlas de los escarabajos de México (Coleoptera: Lamellicornia: Scarabaeidae, Trogidae, Passalidae y Lucanidae)Vol. II (4). 227 pp.

- 2005. Notes on Bolboceratini from Northeastern México with description of a new Bolbocerosoma Schaeffer (Coleoptera: Scarabaeidae: Geotrupinae). Folia Entomológica Mexicana. 44 (2): 213-218.

Lobo, J.M., A. Baselga, J. Hortal., A. Jiménez-Valverde, \& J.F. Gómez. 2007. How does the knowledge about the spatial distribution of Iberian dung beetle species accumulates over time? Diversity and Distributions (in press). doi:10.1111/j.1472-4642.2007.00383.x

Martín-Piera, F., \& J. I. López-Colón. 2000. Coleoptera, Scarabaeoidea I. Fauna Ibérica 14. Museo Nacional de Ciencias Naturales, CSIC, Madrid, Spain.

Martínez-Meyer, E. 2005. Climate change and biodiversity: some considerations in forecasting shifts in species potential distributions. Biodiversity Informatics. 2: 42-55.

Nix, H. A. 1986. A biogeographic analysis of Australian elapid snakes in R.C. Longmore (ed). Atlas of Australian elapid snakes. Australian Flora and Fauna Series No. 7: 4-15. Australian Government Publishing Service, Canberra. 
Peterson, A.T. 2006. Uses and requirements of ecological niche models and related distributional models. Biodiversity Informatics. 3: 59-72.

Peterson, A. T. \& A. Navarro-Sigüenza, H. Benítez-Díaz. 1998. The need for continued scientific collecting: a geographic analysis of Mexican bird specimens. Ibis. 140: 288-294.

Phillips, S. J., R. P. Anderson \& R. E. Schapire. 2006. Maximun entropy modeling of species geographic distributions. Ecological Modelling. 190: 231-259.

Pulliam, H. R. 2000. On the relationship between niche and distribution. Ecology Letters. 3: 349-361.

Reutter, B.A., V. Helfer, A.H. Hirzel \& P. Vogel. 2003 Modelling habitat-suitability using museum collections: an example with three sympatric Apodemus species from the Alps. Journal of Biogeography. 30: 581-590.

Romo, H., E. García-Barros \& J.M. Lobo. 2006. Identifying recorder-induced geographic bias in an Iberian butterfly database. Ecography. 29(6): 873-885.

Soberón, J. \& A.T. Peterson. 2005. Interpretation of models of fundamental ecological niches and species' distributional areas. Biodiversity Informatics. 2: 1-10

Trotta-Moreu, N., E. Montes de Oca \& M. Martínez. 2007. Ecological and reproductive characteristics of Geotrupes (Halffterius) rufoclavatus Jekel, 1865 (Coleoptera: Scarabaeoidea: Geotrupinae) on the Cofre de Perote Volcano (Veracruz, Mexico). Coleopterists Bulletin 61(3): 435-446.

Vaughan, I.P. \& S.J. Ormerod. 2005. The continuing challenges of testing species distribution models. Journal of Applied Ecology. 42: 720-730.

Verdú, J. R., E. Galante, J. P. Lumaret \& F. J. Cabrero-Sañudo. 2004. Phylogenetic analysis of Geotrupidae (Coleoptera, Scarabaeoidea) based on larvae. Systematic Entomology. 29: 509-523.

Zaniewski, A.E., A. Lehmann \& J.M. Overton. 2002. Predicting species spatial distributions using presence-only data: a case study of native New Zealand ferns. Ecological Modelling. 157: 261-280.

Zunino, M. 1984. Sistematica genérica dei Geotrupinae (Coleoptera, Scarabaeoidea: Geotrupinae) filogenesi della sottofamiglia e consideración biogeografiche. Bolletino del Museo Regionale di Scienze Naturale di Torino. 2:9-162. 
